\title{
An Evidence-Based Approach to the Prevention of Oral Diseases
}

\author{
A.J. Spencer \\ Social and Preventive Dentistry, The University of Adelaide, Adelaide, S.A., Australia
}

\section{Key Words}

Evidence-based · Prevention · Oral diseases

\begin{abstract}
The evidence-based approach has become the mantra of health care and service delivery. But just what it means, whether it is feasible, how to build it and the outcome of its use are not well understood. The aims of this paper are to provide an overview of an evidence-based approach to the prevention of oral disease, to examine the assessment of clinical trial evidence, to examine emerging approaches to assessing population-wide interventions and oral health promotion, and to illustrate some principles and issues through examples from preventive dentistry. The evidence-based approach to prevention is presented using an evidence loop, which emphasizes that the evidence-base should begin with an understanding to the burden of oral disease and its determinants, rather than a consideration of the efficacy or effectiveness of interventions in clinical dental research. A systematic review of evidence from clinical dental research is compiled and assessed, after which the intervention is decided upon and implemented. The evidence loop is completed by the monitoring of outcomes and reassessment of the intervention process. Attention is also given to steps in assessing non-randomized population-wide interventions and evidence on oral health promotion based on expert opinion. The requirement for evidence
\end{abstract}

creates a substantial challenge which can only be met by increased research activity, improved quality of information and the appropriate application of the outcomes of research to policy making for the prevention of oral disease.

Copyright $@$ 2003 S. Karger AG, Basel

\section{Introduction}

The evidence-based approach has become the mantra of health care and service delivery. It includes all aspects of dentistry, not the least prevention. But just what it means, whether it is feasible, how to conduct it and the outcome of its use are not well understood. The evidencebased approach to the prevention of oral disease relies on knowledge of the effectiveness of identical, similar, or analogous interventions usually carried out and evaluated in a different setting at a different time. Toward the end of the 1990s some journals published systematic reviews and meta-analyses, or evidence-bases, using quantitative scientific methods and consulting scholars around the world about specific methods of appraising and quantifying the benefits and risks of interventions. However, it was found that outside a few areas of health care, clinical trial evidence is scarce, particularly in many areas of dentistry. Many everyday decisions on health care, including prevention of oral diseases, are based on public health programs and policies founded on less scientific evidence

\section{KARGER \\ Fax +4161306 1234 \\ E-Mail karger@karger.ch \\ www.karger.com

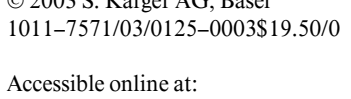

Prof. A. John Spencer

Social and Preventive Dentistry

The University of Adelaide

Adelaide, SA 5005 (Australia)

Tel. +61 88303 5438, Fax +61 88303 4858, E-Mail john.spencer@adelaide.edu.au 
Fig. 1. An evidence loop for the prevention of oral diseases.

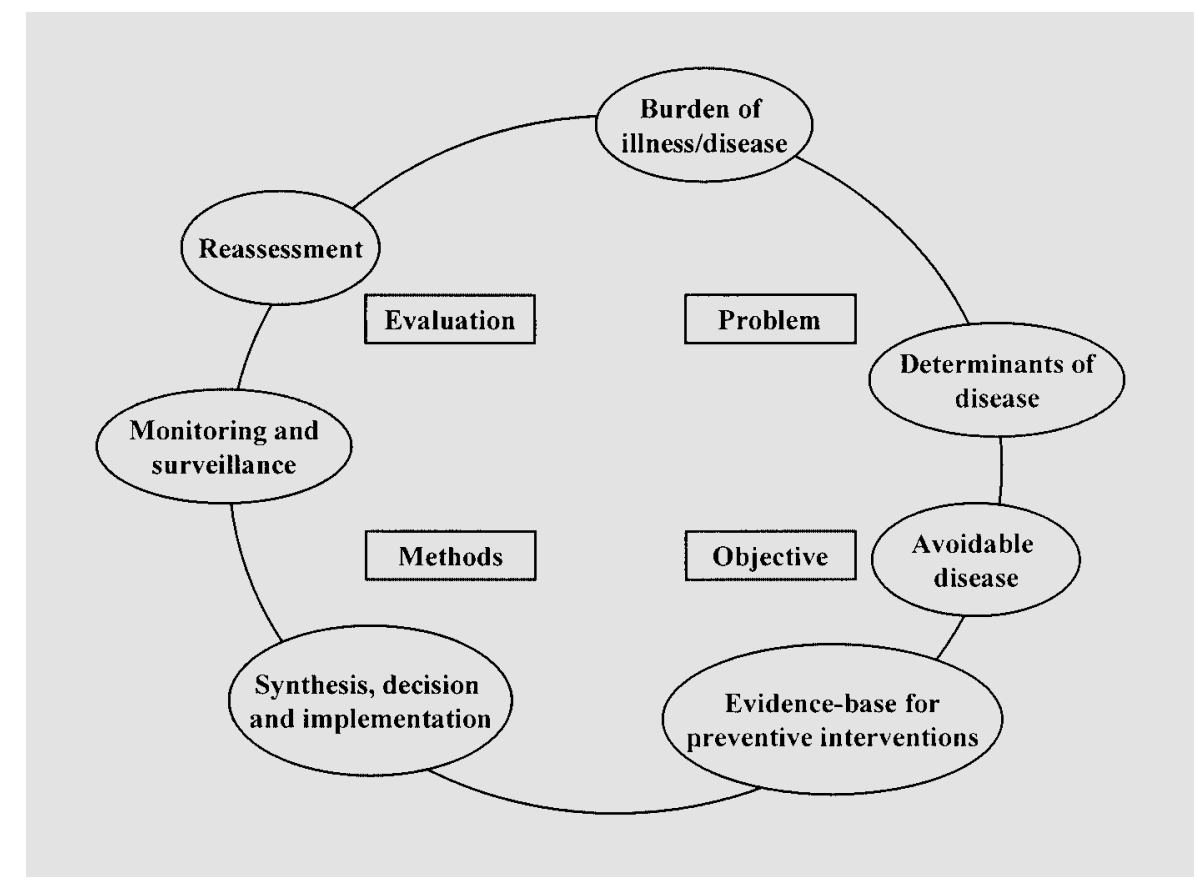

than is required or desired. Not only is more evidence needed, but new ways of examining population-wide interventions and programs for oral health promotion are also needed to assist decision-making.

A number of levels of evidence and methods to assess them are being developed. New concepts for an evidencebased approach and a range of old and new methods for the assessment of evidence seem to be gaining greater clarity. This paper provides an overview of the evidencebased approach to prevention, and points out some of the limitations to applying evidence to population-wide interventions and some issues in oral health promotion.

\section{Evidence-Based Approach to Oral Disease Prevention}

The evidence-based approach to prevention begins with the identification and definition of an oral health problem for which an objective for oral health gain can be stated. Related evidence on the efficacy of interventions is synthesized and assessed, after which an intervention plan is decided upon and implemented. Finally, the oral health outcomes among patients or populations are monitored and the whole process reassessed over time. These fundamental components might be expanded into a more detailed evidence loop for the prevention of oral diseases, as presented in figure 1. Each aspect of this evidence loop for the prevention of oral diseases is necessary for sound decisions on either an individual or population level. Following the various stages ensures that resources are not used to address less important problems or alter less significant determinants, and that preventive interventions are not maintained beyond their useful life should the burden of disease alter. The loop also recognizes that some interventions might work less satisfactorily in different contexts.

\section{Burden of Oral Disease}

In the evidence loop the problem is first identified, defined and prioritized through information on the burden of oral disease, which is the assessment of the magnitude and impact of oral health problems among patients or populations. To design the appropriate intervention, determinants of the disease are delineated and the level of avoidable disease is assessed - i.e. how much of the disease is due to mutable risk factors and what proportion of the burden of disease is avoidable.

Numerous ways exist to measure the burden of disease. Summary health measures such as Disability-Adjusted Life Years (DALYs) provide a common metric. The DALY was first used in a comprehensive assessment of the global burden of disease and injury in 1990 by the World Bank [1] and has been adopted by the 
World Health Organization to inform health planning [2]. DALYs provide a way to link the cause and occurrence of a disease to both short- and long-term health outcomes, including impairments, functional limitations (disability) and death. One DALY is a lost year of 'healthy' life. DALYs are a combination of years of life lost (YLL) due to premature death and equivalent years of life lived with disability (YLD).

Such population-wide 'summary health measures' have been emphasized recently in the development of health policies. A report on the burden of disease and injury in Australia [3] identified oral disease as one of the top dozen major disease groups for non-fatal burden of disease. While mental and nervous system disorders were of substantially higher burden than any others, oral disease ranked in a group of diseases/disorders that are considered highly preventable, such as injuries and infectious diseases. The oral diseases included were dental caries, periodontal disease and subsequent edentulism. Years of life lived with disability were predominantly linked to dental caries (56.2\%), then to periodontal disease (30.3\%) and finally to edentulism (13.5\%). Young and middleaged adults experienced more years of life lived with disability from dental caries than did older adults, while the years of life lived with disability from periodontal disease were distributed among middle-aged adult groups.

The main challenge of using summary health measures is ensuring that the burden of disease is appropriately estimated, so current estimates of the burden of oral disease in DALYs require further consideration. Estimates of the incidence of new disease from cross-sectional prevalence data are not entirely reliable, because the assumptions made in the translation of prevalence to incidence data do not recognize the recurrence of the most common oral diseases (dental caries and periodontal disease) at previously affected sites. Furthermore, the estimates for the amount of disability associated with each oral disease need scrutiny. The summary health measures cited above apply a system of averaged levels of disability, handicap, mental wellbeing, pain and cognitive impairment using a modified version of the EuroQoL health status instrument; by these measures, the disability weights for gingivitis and dental caries were the lowest of all diseases or disorders [4], indicating that the weights need further investigation.

Research using generic quality of life measures among dental patients has shown a low level of impacts; however, this type of research will help assess the relative weightings ascribed to common oral diseases. Orally specific measures of quality of life show a greater sensitivity for oral impacts than do measures for general quality of life
[5], and they have been developed to identify those oral diseases of greater burden that should be considered as targets for prevention [6].

An evidence-based approach to prevention, therefore, requires knowledge of the relative burden of disease associated with particular oral diseases at different stages of life and the proportion of that burden of disease that is avoidable given associations with mutable determinants of disease.

\section{Determinants of Oral Disease}

Preventive programs should be based on conceptual and empirical evidence of the determinants of variation in oral disease among patients or population groups in order to identify more points of intervention in the prevention of oral disease. The conceptual model illustrated in figure 2 identifies three discrete yet closely interrelated stages or levels of determinants: upstream, midstream, and downstream [7].

Upstream level factors: The framework identifies social, physical, economic and environmental factors as being the most fundamental determinants of oral health. These include a range of interrelated factors such as education, employment, occupation, working conditions, income, housing, and area of residence. The framework also indicates that these fundamental determinants are themselves influenced by even more upstream factors, namely, government policies, globalisation, and culture.

Midstream level factors: Social, physical, economic and environmental contexts throughout life influence health either indirectly via psychosocial processes and dental health behaviours, or more directly, for example via injuries. The dental care system also plays some part in determining oral health within a society. However, it plays only a modest and moderating role.

Downstream level factors: Ultimately, oral diseases are a consequence of adverse biological reactions to changes or disruptions in various physiological systems. The poorer health profile of some patients or population subgroups is due in part to longer-term adverse physiological and biological changes that are brought about by poorer psychosocial health and more harmful dental health behaviours.

The concept of 'avoidable oral disease' is based on an understanding of these wider determinants for most oral diseases and the evidence-base for the effectiveness of possible interventions. Three issues at the centre of new approaches to prevention are multifactorial causes of chronic (including oral) disease, shared risk factors, and life stages. It may be more effective and efficient to build 


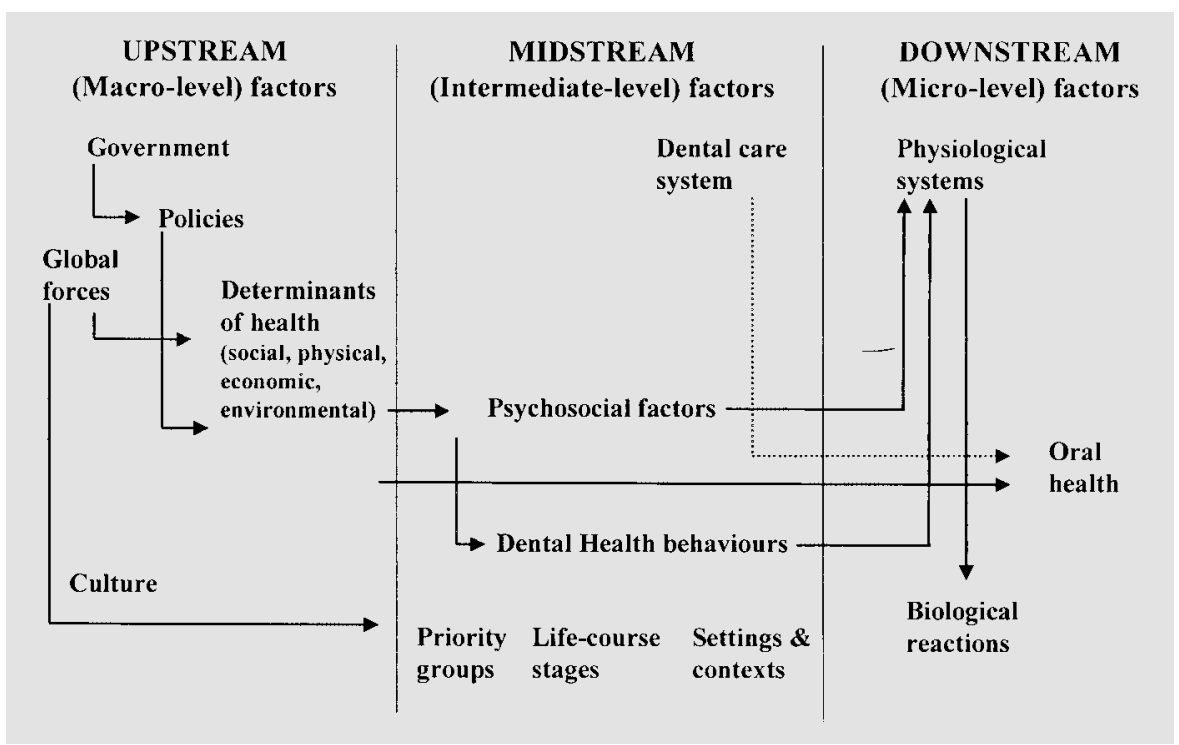

Fig. 2. Determinants of oral disease.

preventive efforts around common risk factors than to develop separate preventive programs for each disease. Activities to prevent many of the risk factors may be undertaken in common settings, such as schools or health centres.

Current knowledge suggests that oral health outcomes are likely to be best when prevention is promoted throughout life (beginning with the prenatal period and infancy and extending through childhood, adolescence, adulthood and older adulthood), because risks and preventive factors accumulate and interact over a lifetime in a dynamic process. The principles, approaches and messages of health promotion (e.g. empowerment, equity, health literacy, healthy behaviours, supportive environments) and specific preventive interventions are relevant throughout a lifetime, but each life stage also has unique contextual and behavioural aspects, and therefore particular strategies to reduce risk factors and strengthen prevention are needed.

Integrated models are emerging that address the continuum of opportunities for prevention, such as the one presented in figure 3, which was developed for Australia's chronic disease strategy [8]. In such models people are distributed across different target groups: the well population, those at risk, those diagnosed with disease, and those with controlled disease. Interventions are specific to these stages and have different objectives, such as preventing movement into the at-risk group, preventing progression to established disease, or averting recurrence of disease and loss of oral function. In such as approach to pre- vention, the evidence-base on different interventions is a key component of the support systems.

\section{Synthesis of the Evidence-Base for Preventive \\ Interventions and Decision-Making}

The evidence-based approach makes use of evaluative research on the effects of an intervention to determine the likely benefits or adverse consequences of intervention for particular individuals or populations. When possible, evidence of beneficial outcomes, rather than biological plausibility or anticipated effects, is used [9]. Evidence of benefits is derived predominantly from epidemiologic research, which provides quantitative estimates of efficacy or effectiveness. Summary estimates of effectiveness are generated by a critical review of research data from two or more studies using systematic review methods [10]. Subgroup analyses may be used to identify characteristics of people for whom an intervention is most or least effective.

The starting point for the traditional evidence-based approach, therefore, is the searching for and collation of the scientific evidence on a given intervention. Questions concerning the intervention should be considered carefully and in detail. Narrow rather than broad questions assist the systematic review of evidence, but the question must still be likely to support practical and potentially useful interventions given favourable evidence.

Considerable emphasis is placed on the transparency and reproducibility of the literature search. Finding studies relevant to an intervention is not easy; beyond sifting 


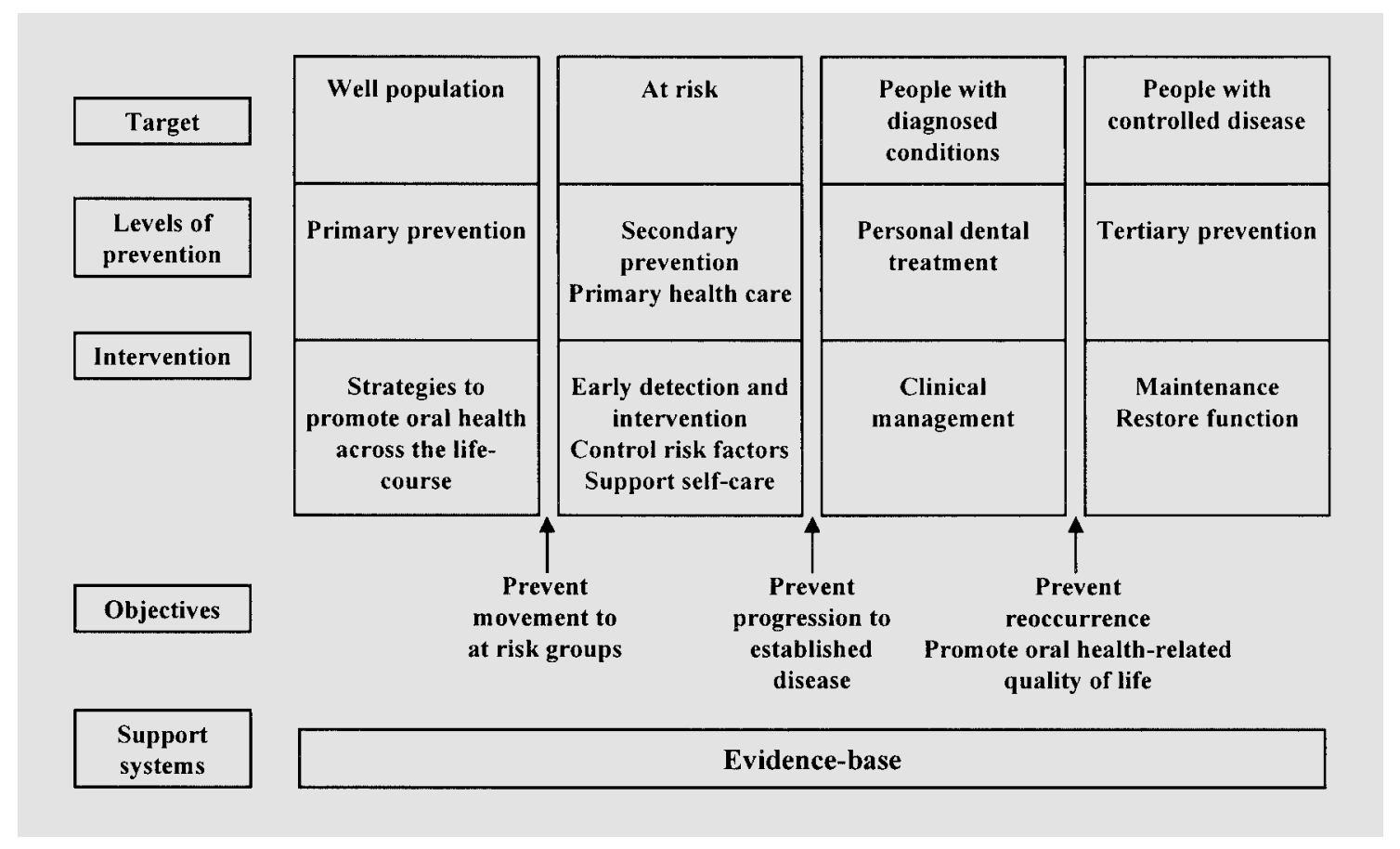

Fig. 3. An integrated model for the opportunities for prevention of oral diseases.

through a mass of literature, there are problems of duplicate publications and accessing the 'grey literature' such as conference proceedings, reports, theses and unpublished studies, as well as the new web-based literature which is growing exponentially. As an initial step it is helpful to find out if a systematic review has already been done. If not, published original articles need to be found through searches of databases using very explicit criteria for inclusion/exclusion in the review. Bibliographies of identified studies can lead to further relevant studies, and hand searching and writing to experts are also essential. The fate of all identified studies needs to be tracked, whether included or excluded in the review.

Relevant studies are then summarized and the research appraised. Numerous guides are available to assist the process of abstracting information from selected studies and putting them in evidence table formats. Many research publications, however, fail to include all the information sought; this could be addressed by adhering to a minimum set of information items that could reasonably be expected in research publications, such as those suggested by the CONSORT statement [11].

Once the review is compiled, evidence is assessed to determine the validity, reliability and precision of the estimates of efficacy of the preventive intervention as well as the size, importance and relevance of beneficial effects, according to the following criteria:

- Strength of evidence: was the research good enough to support a decision on whether or not to implement an intervention?

- Size of the effect: what were the research results?

- Relevance: do they apply to the potential recipients of the preventive intervention [12]?

The level of evidence indicates the validity of evaluative research and takes into account the design of the study, its potential for eliminating bias, and the methods and analysis used [13]. An example of a classification of the level of evidence based on study design is presented in table 1 .

As an illustration, we will apply the three above-mentioned assessment issues (strength of evidence, size of the effect, and relevance) to the evidence-base for an oral health preventive intervention: fissure sealants performed in a clinic-based dental program for school children. Fissure sealants have been the subject of much clinical trial research. The level of evidence is high because the clinical intervention can be randomly assigned to either children or one of a contralateral pair of teeth, eliminating bias and, with appropriate statistical testing, chance from outcomes. 
Table 1. Level of evidence and study design

\begin{tabular}{ll}
\hline Level of evidence & Study design \\
\hline I & $\begin{array}{l}\text { Evidence obtained from a systematic review } \\
\text { of all relevant randomized controlled trials }\end{array}$ \\
\hline II & $\begin{array}{l}\text { Evidence obtained from at least one } \\
\text { properly designed randomized controlled } \\
\text { trial }\end{array}$ \\
\hline III-1 & $\begin{array}{l}\text { Evidence obtained from well-designed } \\
\text { pseudo-randomized controlled trials } \\
\text { (alternate allocation method) }\end{array}$ \\
\hline III-2 (observational) & $\begin{array}{l}\text { Evidence obtained from comparative } \\
\text { studies with concurrent or historical } \\
\text { control groups, cohort studies, case- } \\
\text { control studies or interrupted time-series } \\
\text { with a control group }\end{array}$ \\
\hline III-3 (comparative) & $\begin{array}{l}\text { Evidence obtained from comparative } \\
\text { studies with historical control }\end{array}$ \\
\hline IV & \begin{tabular}{l} 
Evidence obtained from case series \\
\hline Excluded
\end{tabular} \\
\hline $\begin{array}{l}\text { Evidence from expert opinion and } \\
\text { consensus of an expert committee }\end{array}$ \\
\hline
\end{tabular}

Source: [14].

The size of the effect has been expressed variously as rates of retention of sealants over time or statistically significant reductions in dental caries increment. Other research has highlighted the intriguing potential for sealants to prevent caries on adjacent non-sealed surfaces, indicating that the effect may be larger than the prevention of dental caries on sealed pit and fissure surfaces [15]. However, there were more studies on the retention of sealants than on caries increment, raising questions on the appropriateness and relevance of outcomes. Retention is not the same as caries prevention [16].

In this example, it should be pointed out that different studies may have used different measures of effect. Clearly, only studies using the same measures are comparable. It must be decided if studies using different measures will be grouped together or if the type of effect measurement will be a criterion for including the study in the evidencebase or excluding it. Furthermore, the quality of individual studies can vary even within a single level of evidence, according to the study design; to address this potential drawback, a quality score can be given to each study based on methodological features like randomization, blinding, and retention of subjects. Since this is a subjective judg- ment, most systematic reviews are based on quality scores given by two or more individuals. The level of agreement among assessors needs to be reported.

After the research is appraised, the next stage is the synthesis, or pooling, of the evidence. While this might take a qualitative approach with some overview statement, most often quantitative methods like meta-analysis are used. Meta-analysis relies upon the similarity of studies and increases the power and generalizability of effects. Analyses vary depending on the type of effect measurement used (binary or continuous) and whether it is independent or paired (as in many fissure sealant trials), as well as on the sensitivity of the analysis and the potential for publication bias. Sensitivity can be tested by analysing studies that are rated at different levels of evidence separately and comparing results. Another way to test sensitivity is to categorize by quality score those studies that fall within a single level of evidence; this way, studies in different categories of quality score can be analyzed separately, or studies in lower quality categories sequentially added to the analysis and the results compared. A number of discrete approaches exist to examining publication bias including funnel plots and regression asymmetry.

Once the evidence is synthesized, decisions must be made about how to apply the evidence, taking into consideration the transferability of outcomes to patients or population groups and the predicted effects of implementing the intervention. With regard to transferability, both the beneficial and harmful effects of an intervention need to be considered in the collective group of patients and different subgroups among those patients. Baseline risk of disease must also be taken into account. To apply the evidence to individuals, absolute benefits in target populations are predicted, and it is decided whether predicted benefits outweigh any predicted harm.

While some guides to synthesis and decision-making end here, the evidence-based approach to the prevention of oral diseases includes a number of other important issues, such as considerations of efficiency, public perceptions and side effects. Efficiency is determined by the relationship between the resources used and the outcome; it includes economic analyses such as cost-effectiveness or cost-benefit analyses. These techniques are also important aspects of the evidence-base for prevention [17]. Perceptions of the public and side effects are covered briefly in later sections.

\section{Implementation, Monitoring and Reassessment}

Once a decision on the appropriate oral health preventive intervention has been made, based either on a sys- 
tematic review of randomized clinical trials or an assessment of observational studies, the intervention is implemented. The last stage of the evidence loop is the monitoring of patients or population groups and the reassessment of the value and necessity of continuing the intervention. While the importance of this final stage is readily acknowledged, all too frequently resources and energy are expended on the intervention and little effort goes into monitoring the outcome. Without this reassessment at the local level, questions on whether a program should continue or be modified will be inadequately addressed.

\section{Limitations of the Rules of Evidence Applied to Population-Wide Interventions}

Recently, a number of limitations to the classic appraisal and application of evidence for preventive interventions have come under scrutiny $[18,19]$. Most public health, population-wide preventive interventions are programmatic in nature, covering numbers of people in defined areas. But studies are rarely conducted on such interventions, and when they are, they are observational studies and not randomized. Evidence derived from observational research is considered to be of a lower level because of its potential for bias. However, this devaluation of observational studies emphasises the issue of bias and fails to recognize the importance of transferability. Randomized clinical trials also have drawbacks because they are conducted among unrepresentative samples of the population. In addition, recruitment to a trial is often associated with greater compliance with the intervention than might be reasonably expected in the population, leading to the distinction between a randomized clinical trial and a community trial [20]. Furthermore, a randomized clinical trial takes place over a shorter period, possibly masking either a decrease in efficacy over the longer term or the emergence of side effects.

These difficulties with the accepted hierarchy of evidence are illustrated by the issue of water fluoridation. While cluster randomized controlled trials of water fluoridation could be designed in theory, studies of this type are unknown. Instead, evidence on water fluoridation is generally derived from observational studies of discrete geographic areas with and without fluoridation, before and after the water fluoridation was introduced. While these designs may have been the most feasible, acceptable and appropriate [21], they are considered to be at a lower level in the evidence hierarchy [22]. The issue that emerges is how to view a preventive measure where there is a large number of studies which are all individually at a lower level of evidence. A recent report argued that because of the number of studies, the level of evidence should be regarded as higher than that indicated by their study design alone [23].

Another interesting evidence issue illustrated by the case of water fluoridation is the public's perception of the intervention. An intervention will only be implemented if there is general public support for its application. Despite the successful implementation of water fluoridation programs in many countries, the public still does not know much about it and expresses occasional concern about its safety, while a sizeable minority of the population may be undecided or opposed to it [24, 25]. Therefore, public perception may assist or impede the implementation of an evidenced-based preventive intervention, ultimately determining whether the community will benefit from the measure.

\section{Evidence Issues in Oral Health Promotion}

Health promotion programs which aim to improve oral health often promote a mix of interventions, or a 'portfolio' [19]. These may be effective, but they do not fit well the requirements for evidence of effectiveness. Also important is the context or setting of interventions and how it may shape the outcome.

For example, an oral health promotion program might be built around existing 'healthy' baby activities such as antenatal and parent education and well-baby and immunization checks [26, 27]. There is an emerging interest in this oral health promotion opportunity, but little research has been conducted [28]. Interventions may be adopted that are a combination of approaches that represent current 'best practice' in health education, behavioural change, avoidance of common risk factors, and monitoring of health care providers. Recent reviews of health promotion for oral health have evaluated such oral health promotion interventions and adjusted the thresholds for levels of evidence, specifically including expert opinion and influential reports as a low but acceptable level of evidence. They also weighed the revised levels of evidence against the potential benefit for oral health [23]. The potential benefit can be classified as one of the following:

- beneficial;

- likely to be beneficial;

- trade-off between beneficial and adverse effects;

- unknown;

- unlikely to be beneficial, and

- likely to be ineffective or harmful [23]. 
A practical guide for decision-makers to use in selecting a portfolio or mix of interventions for oral health promotion has recently been proposed as part of health promotion planning and practice improvement [19]. Unlike the approaches required for scientific, quantitative evidence, such frameworks hope to ensure that the best available evidence, knowledge and expertise are brought to bear on the problem at hand and that the portfolio ensures a comprehensive approach to addressing the problem.

The distinct steps of the portfolio approach are:

- convene a decision-making group;

- specify the issue to be addressed;

- agree to the criteria against which to judge interventions;

- weigh the criteria to be used to evaluate options;

- brainstorm a long-list of interventions likely to fulfil the criteria;

- specify a short-list of interventions for more detailed evaluation;

- evaluate the short-listed interventions against the weighted criteria;

- score and prioritise the interventions;

- reflect on the outcomes of the exercise and refine if necessary [19].

While such an approach uses the best evidence available, the disadvantages of these portfolios of interventions are that they can never disentangle their component effects, or might not be open to falsification.

\section{Conclusion}

Although the evidence-based approach is 30 years old, just what it means, whether it is feasible, how to conduct it and the outcome of its use are not well understood. This situation holds in dentistry in general and in areas like the prevention of oral disease in particular. In order to present a comprehensive evidence-based approach to the prevention of oral diseases, an evidence loop has been presented. The evidence for the prevention of oral diseases begins with an understanding of the burden of oral disease at different life stages and the proportion which is avoidable, given associations with mutable determinants of disease. This provides a broad underpinning health policy and priority setting giving direction to both individual and population-wide preventive interventions. The key questions to be addressed about those interventions focus on the beneficiaries, efficacy, efficiency, public perceptions and side effects.
A systematic review of the literature is a key component of the evidence-based approach. Guidance is available for the searching, selecting, abstracting and appraising, synthesis and decision-making on clinical trial evidence. While these guidelines are readily applicable to clinical interventions, difficulties arise in the areas of population-wide interventions and oral health promotion, as exemplified by the issue of water fluoridation where randomization is not feasible, but lower level evidence is available from community trials. Further difficulties arise in the area of oral health promotion where portfolios of interventions are common. More recently evidence-based processes have emerged that are more appropriate for these later situations.

Regardless of the level of evidence or approaches to its appraisal, the evidence loop is completed by implementation, monitoring and reassessment. Too frequently an intervention for which evidence has been found beneficial is inadequately reassessed over time in target patients or populations, but without reassessment it is difficult to determine the value and necessity of maintaining a given intervention. All of the stages of the evidence-based approach are very important and taken together, they offer a rational way forward to improve oral health and dental care.

\section{Acknowledgement}

This paper is based on a presentation at the 7th World Congress on Preventive Dentistry April 24-27, 2001, Beijing, China. 


\section{References}

1 World Bank: World Development Report 1993: Investing in Health. New York, Oxford University Press, 1993.

2 World Health Organization: The World Health Report 1999. Geneva, WHO, 1999.

3 Mathers C, Vos T, Stevenson C: The Burden of Disease and Injury in Australia. Canberra, Australian Institute of Health and Welfare, 1999.

4 Stouthard M, Essink-Bot M, Bonsel G, Barendregt J, Kramers P: Disability weights for diseases in the Netherlands. Rotterdam, Department of Public Health, Erasmus University, 1997.

5 Cunningham SJ, Garratt AM, Hunt NP: Development of a condition-specific quality of life measure for patients with dentofacial deformity. II. Validity and responsiveness testing. Community Dent Oral Epidemiol 2002;30:8190.

6 Slade GD (ed): Measuring Oral Health and Quality of Life. Chapel Hill, Dental Ecology, University of North Carolina, 1997.

7 Turrell G, Oldenburg B, McGuffog I, Dent R: Socio-Economic Determinants of Health: Towards a National Research Program and a Policy and Intervention Agenda. Brisbane, School of Public Health, Queensland University of Technology, 1999

8 National Public Health Partnership: Preventing Chronic Disease: A Strategic Framework. Background Paper. Melbourne, NPHP, 2001.

9 Chalmers I: What do I want from health research and researchers when I am a patient? BMJ 1995;310:1315-1318.

10 Mulrow CD, Oxman AD (eds): Cochrane Collaboration Reviewers Handbook; in: The Cochrane Library. The Cochrane Collaboration. Oxford, Update Software, 1999. Accessed online: http://www.update.software.com/ccweb/ cochrane/hbook.htm.
11 Begg C, Cho M, Eastwood SW, Mŏher D, Olkin I, Pitkin R, Rennie D, Schulz KF, Simel D, Stroup DF: Improving the quality of reporting of randomized controlled trials: The CONSORT statement. JAMA 1996;276:637-639.

12 Oxman AD, Sackett DL, Guyatt GH: Users guides to the medical literature. 1. How to get started: The evidence-based medicine working group. JAMA 1993;270:2093-2095.

13 Sackett DL, Richardson WS, Rosenberg W, Haynes RB: Evidence-Based Medicine: How to Practice and Teach EBM. New York, Churchill-Livingstone, 1997.

14 National Health and Medical Research Council: A Guide to the Development, Implementation and Evaluation of Clinical Practice Guidelines. Canberra, NHMRC, 1999.

15 Bravo M, Baca P, Llodra JC, Osovio E: A 24month study comparing sealant and fluoride varnish in caries reduction on different permanent first molar surfaces. J Public Health Dent 1997;57:184-186.

16 Deery C, Fyffe HE, Nugent ZJ, Nuttall NN, Pitts NB: A proposed method for assessing the quality of sealants - the CCC Sealant Evaluation System. Community Dent Oral Epidemiol 2001;29:83-91.

17 Niessen LC, Douglass CW: Theoretical considerations in applying benefit-cost and cost-effectiveness analyses to preventive dental programs. J Public Health Dent 1984;44:156168.

18 Rychetnik L, Frommer M: A Proposed Schema for Evaluating Evidence on Public Health Interventions. Melbourne, National Public Health Partnership, 2000.

19 National Public Health Partnership: Deciding and Specifying an Intervention Portfolio. Melbourne, NPHP, 2001.
20 O'Mullane DM: Efficiency in clinical trials of caries preventive agents and methods. Community Dent Oral Epidemiol 1976;4:190-194.

21 Black N: Why we need observational studies to evaluate the effectiveness of health care. $\mathrm{Br}$ Med J 1995;312:1215-1218.

22 Irwig LM, Cumming C: Study types in population health research; in Leeder S, Wigglesworth E (eds): Research on Health in Human Populations. Melbourne, The Menzies Foundation, 1988, Menzies Technical Reports No 3, pp 39_ 54

23 Wright FAC, Satur J, Morgan MV: EvidenceBased Health Promotion. Resources for Planning. 1. Oral Health. Melbourne, Health Development Section, Public Health Division, Department of Human Services, 2000.

24 Spencer AJ, Slade GD, Davies MJ: Water fluoridation in Australia. Community Dent Health 1996;13:27-37.

25 Spencer AJ, Stewart JF: Support for water fluoridation in Australia. J Dent Res 1997;76:394, abstr 3044.

26 Jones CM, Tinanoff N, Edelstein BL, Schneider DA, DeBerry-Summer B, Kanda MB, Brocato RJ, Blum-Kemelar D, Mitchell P: Creating partnerships for improving oral health of low-income children. J Public Health Dent 2000;60:193-196.

27 Tinanoff N, Palmer CA: Dietary determinants of dental caries and dietary recommendations for pre-school children. J Public Health Dent 2000;60:197-206.

28 Gunoy H, Dmoch-Bockhorn K, Gunay Y, Geurtsen W: Effect on caries experience of a long-term preventive program for mothers and children starting during pregnancy. Clin Oral Invest 1998;2:137-142. 